\title{
The role of spatial frequency in the processing of hierarchically organized stimuli
}

\author{
MARVIN R. LAMB and E. WILLIAM YUND \\ Veterans Administration Medical Center, Martinez, California
}

\begin{abstract}
Can spatial frequency differences between local and global forms account for differences in the way different levels of structure are analyzed? We examined this question by having subjects identify local or global forms of hierarchical stimuli that had been contrast balanced. Contrast balancing eliminates low spatial frequencies, so that both local and global forms must be identified on the basis of high spatial frequency information. Response times (RTs) to global (but not local) forms were slowed for contrast-balanced stimuli, suggesting that low spatial frequencies mediate the global RT advantage typically found. In contrast, interference between local and global forms was little affected by contrast balancing or by shifts of attention between local and global forms, suggesting that it does not result from inhibitory interactions between spatial frequency channels or from temporal precedence of low versus high spatial frequency information. Finally, shifts of attention between local and global forms were also little affected by contrast balancing, suggesting that they were not based on spatial frequency.
\end{abstract}

Campbell and Robson (1968) were among the first to suggest that there might be a set of channels in the visual system, each responsive to its own restricted range of spatial frequencies. There is now ample evidence, both physiological and psychophysical, for the existence of such channels (see R. L. De Valois \& K. K. De Valois, 1990, for a comprehensive review). Several investigators have suggested that the analysis of local versus global levels of structure might depend on the differences in spatial frequency between local and global forms (Badcock, Whitworth, Badcock, \& Lovegrove, 1990; Hughes, 1986; Hughes, Fendrich, \& Reuter-Lorenz, 1990; Lamb \& Robertson, 1990; Navon, 1991; Sergent, 1982, 1987; Shulman, Sullivan, Gish, \& Sakoda, 1986). The identity of global forms (e.g., the " $A$ " in the upper left of Figure 1) is specified by relatively low spatial frequencies, whereas the identity of local forms (e.g., the " $S$ "'s in the upper left of Figure 1) is specified by relatively high spatial frequencies. Thus, differences in the ability to identify local and global forms might result from differences in the functional characteristics of high versus low spatial frequency channels.

Several studies have provided support for this view. Shulman et al. (1986) adapted subjects to sinusoidal grat-

This research was supported by the Medical Research Council of the Veterans Administration and by National Institute of Neurological Disorders and Stroke Grant NS27902 to M. R. L. We would like to thank Lester Krueger, John Uhlarik, and an anonymous reviewer for many insightful comments. We are also very grateful to Russell $L$. De Valois for allowing us to use his equipment to calculate the Fourier transforms of our stimuli. Correspondence concerning this article should be addressed to M. R. Lamb, Research 151, Veterans Administration Medical Center, 150 Muir Road, Martinez, California 94553 (e-mail: marva4! marvin@ucdavis.edu). ings of different spatial frequencies, and then had them identify local or global forms. They found that the adapting frequency most affecting global identification was lower than the adapting frequency most affecting local identification. Badcock et al. (1990) used a Gaussian filter to selectively eliminate low spatial frequencies and found that all performance differences between local and global forms were eliminated. These data show that, at the very least, some aspect of the analysis of hierarchically organized stimuli is sensitive to the differences in spatial frequency typically associated with local and global forms.

The present experiments further explore the role of spatial frequency in the analysis of hierarchically organized stimuli by examining whether attentional selection between different levels of structure depends on spatial frequency. Shulman and Wilson (1987) have already provided evidence consistent with this possibility. They induced subjects to attend selectively to different levels of structure by having them identify local or global forms in separate blocks of trials. On a small number of probe trials, the subjects were also asked to detect sine wave gratings of different spatial frequencies. Low-frequency gratings were detected more easily than high-frequency gratings in the context of the global task, whereas the reverse was true in the context of the local task. This is consistent with the notion that attention to level occurred via selection between, or differential weighting of, high versus low spatial frequency channels.

As the authors point out, however, the fact that the change in grating detectability was frequency specific does not necessarily mean that spatial frequency was the basis for selection between the local and the global forms. An alternative is that the area encompassed by an attentional "spotlight" varied depending on whether the task was local or global (Lamb \& Robertson, 1988, 1990; Ward, 


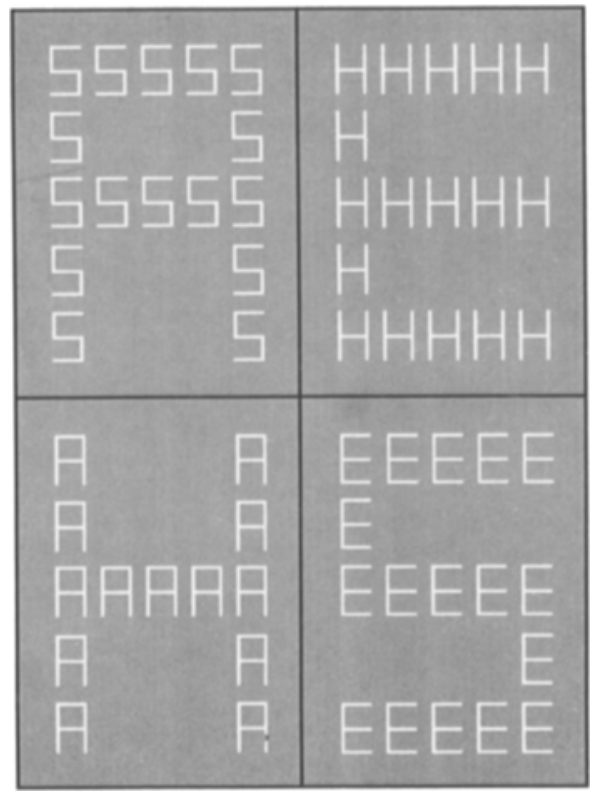

Figure 1. Drawings of four (of the eight) hierarchical letter patterns used in the present experiments. Shown are a global " $A$ " composed of local "S"s (upper left), a global "E" composed of local "H"s (upper right), a global " $H$ " composed of local " $A$ "s (lower left), and a global " $S$ " composed of local " $E$ "s (lower right).

1982). That is, during the local task subjects might have attended to a small region corresponding to the size of a local form, whereas during the global task, a wider area might have been attended. Such a difference in the size of the attended area might then selectively benefit the analysis of high versus low spatial frequencies. For example, too small an attended area might impair the analysis of low spatial frequencies because too few cycles would fall within the attended area. Conversely, a larger attended area should benefit the analysis of low spatial frequencies but might not be optimal for the analysis of high spatial frequencies.

To address this issue, we examined the ability to shift attention between local and global forms for stimuli that had been contrast balanced (Carlson, Anderson, \& Moeller, 1980; Hughes et al., 1990). Contrast balancing selectively eliminates low spatial frequencies so that both local and global forms must be identified on the basis of high spatial frequencies. Thus, contrast balancing should impair attentional shifts between local and global forms if they are based on selection between high versus low spatial frequency channels.

A second goal of the present experiments was to elucidate the mechanisms producing interference between global and local forms. Traditionally, such interference has been assumed to reflect the order in which different levels of structure are analyzed. For example, Navon (1977) presented hierarchically organized stimuli similar to those shown in Figure 1, and found that (1) global forms were identified more rapidly than local ones (i.e., a global response time [RT] advantage), and that (2) local RTs were affected by the identity of the global form but global RTs were not affected by the identity of the local form (i.e., asymmetric global interference). Navon concluded that the global RT advantage occurred because information specifying the correct response was available earlier for global than for local forms. Interference was thought to be asymmetric for the same reason. If global analysis precedes local analysis, global information would be present to interfere with local responding, but little or no local analysis would have occurred at the time the response to global targets occurred. Thus, the co-occurrence of these two events was part of the basis for the global precedence hypothesis, which holds that global levels of structure enjoy a temporal processing advantage.

More recently, attempts have been made to explain interference between local and global forms in terms of the functional characteristics of spatial frequency channels (Hughes, 1986; Hughes, Layton, Baird, \& Lester, 1984; Hughes et al., 1990). For example, Hughes (1986) presented compound gratings consisting of a high- and a lowfrequency sinusoid. He found that the low-frequency component interfered with responses to the high-frequency component, but not vice versa. This asymmetry is reminiscent of the asymmetric global interference typically found with hierarchical stimuli and so is consistent with the idea that asymmetric global interference results from the functional characteristics of spatial frequency channels.

Spatial frequency channels might produce interference effects in either of two ways. First, low-frequency channels might operate more rapidly than high-frequency channels (Breitmeyer, 1975; Breitmeyer \& Ganz, 1976; Harwerth \& Levi, 1978; Hughes et al., 1990). This simply restates the global precedence hypothesis in terms of spatial frequency, and like the global precedence hypothesis, it predicts that interference should vary directly with processing priority. Conditions that produce a temporal advantage for the analysis of low frequencies should produce asymmetric low-frequency interference, whereas conditions that produce a temporal advantage for the analysis of high frequencies should produce asymmetric highfrequency interference. In other words, processing advantage and interference should covary.

The second alternative is that interference results not from a temporal advantage for low spatial frequency information, but from inhibitory interactions between high and low spatial frequency channels (K. K. De Valois, 1977; Hughes et al., 1990; Singer \& Bedworth, 1973; Tolhurst, 1972). This inhibitory-interaction hypothesis attributes asymmetric global interference to active inhibition of high spatial frequency channels by low spatial frequency channels. This hypothesis does not require that low spatial frequency channels enjoy a temporal processing advantage for asymmetric interference to occur. In fact, asymmetric low-frequency interference might be found even under conditions that favor faster analysis of high spatial frequencies. Thus, the inhibitory-interaction hypothesis can accommodate a decoupling of processing 
priority and interference, whereas the precedence hypothesis cannot. In fact, there is growing evidence that RT advantage and interference do not always covary in the manner predicted by the precedence hypothesis (Lamb \& Robertson, 1988, 1989; Lamb, Robertson, \& Knight, 1989, 1990; Navon \& Norman, 1983; Paquet, 1992). For example, Lamb and Robertson (1989) varied the visual angle of hierarchical stimuli similar to those in Figure 1. Like Navon, they found a global RT advantage and asymmetric global interference (both consistent with global precedence) when the visual angle was small, but a local RT advantage (consistent with local precedence) and asymmetric global interference (consistent with global precedence) at larger visual angles. Clearly, RT advantage and interference cannot both reflect precedence in this case. These data are not consistent with the precedence hypothesis but can be accommodated by the inhibitory-interaction hypothesis.

Eliminating low spatial frequencies from the stimulus would seem to provide a fairly direct way of discriminating between these hypotheses. If asymmetric global interference reflects the inhibition of high spatial frequency channels by low spatial frequency channels, it should be eliminated when low spatial frequencies are not present. Furthermore, this should be the case regardless of whether local or global forms enjoy an RT advantage. That is, the inhibitory-interaction hypothesis predicts changes in interference as spatial frequency content changes, but it does not require RT advantage and interference to covary. In contrast, if interference and RT advantage simply reflect precedence, any variable that affects $R T$ advantage should also affect interference, regardless of spatial frequency content. If RT advantage and interference do not covary, these two measures cannot both reflect precedence.

Previous experiments have not been designed with this question in mind, and the data turn out to be somewhat ambiguous on this point. Badcock et al. (1990) found that filtering low spatial frequencies from hierarchically organized stimuli eliminated all interference between local and global forms, a finding consistent with the inhibitoryinteraction hypothesis. However, the typical global RT advantage was also eliminated, so filtering produced a covariation between RT advantage and interference consistent with the precedence view. Hughes et al. (1990) found that eliminating low spatial frequencies resulted in mutual interference between global and the local forms. This interference could not be due to interactions between high and low spatial frequency channels because low spatial frequencies had been eliminated. On the other hand, there was little evidence of covariation between RT advantage and interference in this study, so the precedence hypothesis also received little, if any, support.

One reason for the ambiguity of these experiments with respect to this particular question is that they did not include conditions that produced an absolute reversal in RT advantage. Without such a reversal, it is difficult to clearly assess whether RT advantage and interference have covaried as the precedence view demands. To clarify this issue, we included conditions (i.e., attentional biasing) that do produce absolute reversals of RT advantage. Under these conditions, the two interference hypotheses make very clear predictions. If interference reflects simple precedence, RT advantage and interference should covary, regardless of whether or not the stimuli contain low spatial frequencies. If, on the other hand, interference reflects inhibitory interactions between high and low spatial frequency channels, then eliminating low spatial frequencies should eliminate interference regardless of whether there is a global or a local RT advantage.

\section{EXPERIMENT 1}

The procedure for Experiment 1 was similar to that used previously to study shifts of attention between local and global levels of structure (Kinchla, Solis-Macias, \& Hoffman, 1983; Lamb \& Robertson, 1987; Robertson, Lamb, $\&$ Knight, 1988, 1991). The subjects viewed stimuli similar to those in Figure 1. Each stimulus contained one target letter and one distractor letter. In one block of trials (local bias), the targets were more likely to appear at the local level, and in another block (global bias), the targets were more likely to appear at the global level. The subjects had to identify which target letter was present in the stimulus, regardless of the level at which it appeared. Typically, subjects shift from a global RT advantage in the global bias condition to a local RT advantage in the local bias condition. This performance tradeoff presumably reflects shifts of attention due to changing task demands.

Experiment 1 also included a measure of interference that has been used previously (Lamb \& Robertson, 1989; Lamb et al., 1990). The letters " $H$ "' and " $S$ "' served as targets, and the letters " $A$ " and " $E$ "' served as distractors. As can be seen in Figure 1, " $A$ " and " $H$ " were identical except for the addition of the horizontal top segment in the " $A$," and " $E$ " and " $S$ " were identical except that the lower vertical segment was on the left for the " $E$ " and on the right for the " $S$." With these stimuli, the target " $H$ " is identified faster when the distractor is an " $A$ " than when it is an " $E$," and the target " $S$ " is identified faster when the distractor is an " $E$ " than when it is an "A." It has been asserted that this pattern depends on the specific similarity relations between the various targets and distractors, but this assumption is not important for the present purpose. Here, the important point is that this pattern of performance confirms that forms at one level of structure are affecting responses to forms at the other and thus serves as a measure of interference.

The subjects also received two different types of stimuli. The first type consisted of typical broadband stimuli, with high spatial frequencies carrying information about both local and global forms, as well as low spatial frequencies carrying information about global but not local forms. These stimuli appeared simply as white letters on a gray background (Figures 1 and $2 a$ ) and will be referred 


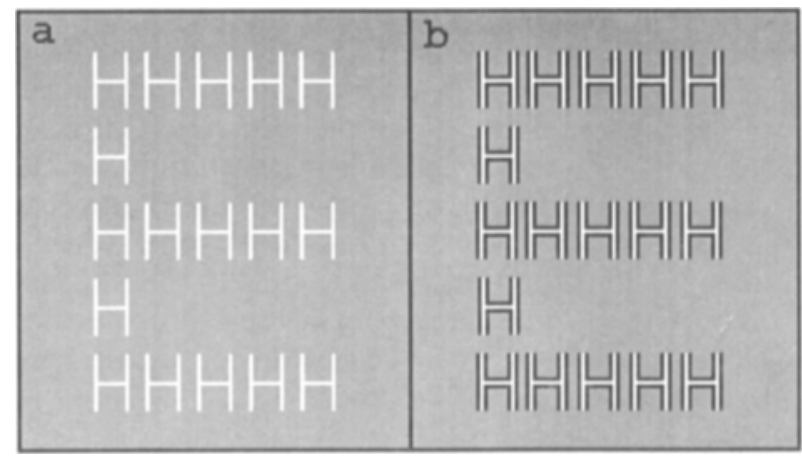

Figure 2. Drawings of a bright stimulus (a) and a contrast-balanced stimulus (b). The precise spatial frequency content of the stimuli depends critically on luminance levels, which are not accurately reproduced in this figure. See text for actual luminance levels. tion of representative bright and contrast-balanced stimuli. Figure $3 \mathrm{a}$ shows the transform for the bright stimulus shown in Figure $2 \mathrm{a}$. As can be seen, bright stimuli contained a broad spectrum of spatial frequencies, with power concentrated near horizontal and vertical orientations. Figure $3 \mathrm{~b}$ shows the transform for the contrastbalanced stimulus shown in Figure $2 \mathrm{~b}$. This figure shows that the contrast-balanced stimuli had much less power at low spatial frequencies than did the bright stimuli. More specifically, the contrastbalanced stimuli had virtually no power at spatial frequencies below 3 cycles/degree (cpd; i.e., the area within the inner circle in Figure 3) and very little power even out as far as $6 \mathrm{cpd}$ (i.e., the area between the two circles in Figure 3$){ }^{1}$

A small $\left(.26^{\circ}\right)$ black $\left(16 \mathrm{~cd} / \mathrm{m}^{2}\right)$ square presented in the center of the screen served as the fixation point. All luminance measurements were taken with a Minolta CS- 100 Chroma Meter. The experiment was conducted with ordinary overhead room lighting.

Procedure. The distance between the subject and the monitor screen was fixed at $65 \mathrm{~cm}$ through the use of a chinrest head re- to as bright stimuli (after Hughes et al., 1990). The second type was identical to the first, except that each local letter was surrounded by a dark outline (Figure $2 b$ ). The luminances of the bright center and dark outline were chosen so that the space-averaged luminance of the compound letter was equal to the luminance of the background. These will be referred to as contrast-balanced stimuli. Contrast balancing eliminates low spatial frequencies that specify global letters. Thus, for contrast-balanced stimuli, both local and global letters must be identified on the basis of high spatial frequencies.

\section{Method}

Subjects. The 5 female and 7 male subjects ranged in age from 18 to 36 years $(M=23.2, S D=5.4)$, were recruited from the staff of the V. A. Medical Center and from a local university, were paid for their participation, gave informed consent, and reported having normal or corrected-to-normal visual acuity. All but one were right handed.

Apparatus. The stimuli were generated on a NEC 4-D color monitor controlled by an 80386-based microcomputer (IBM compatible) and a VGA graphics card. Stimulus timing (onset, termination, and duration) was tied to the vertical sync pulse (refresh rate approximately $60 \mathrm{~Hz}$ ). All other events (responses, intertrial interval [ITI], etc.) were timed with the 8253 chip set to a 1 -msec time base. The status of the response keys (two push-button microswitches of a Gravis game controller) was monitored via the game port.

Stimuli. Global letters were constructed from local letters in a $5 \times 5$ matrix. The letters " $H$ " and " $S$ " served as targets, and the letters " $A$ " and " $E$ " served as distractors. Each stimulus pattern contained one target and one distractor letter, resulting in eight stimuli (four of which are shown in Figure 1). The global letters were $5.1^{\circ}$ high and $3.3^{\circ}$ wide. The local letters were $.71^{\circ}$ high and $.44^{\circ}$ wide.

All stimuli were presented on a gray $\left(46 \mathrm{~cd} / \mathrm{m}^{2}\right)$ background. The bright stimuli (see Figure 2a) were composed of lines that were brighter $\left(66.5 \mathrm{~cd} / \mathrm{m}^{2}\right)$ than the background. The contrast-balanced stimuli (see Figure $2 b$ ) were identical to the bright stimuli, except that the bright lines composing each local letter were surrounded by lines that were darker $\left(36.1 \mathrm{~cd} / \mathrm{m}^{2}\right)$ than the background. The luminance levels of the bright and dark lines were chosen so that the space-averaged luminance of the contrast-balanced stimuli would equal that of the background.

To verify that contrast balancing had, as intended, eliminated low spatial frequencies, we did a two-dimensional Fourier transforma-

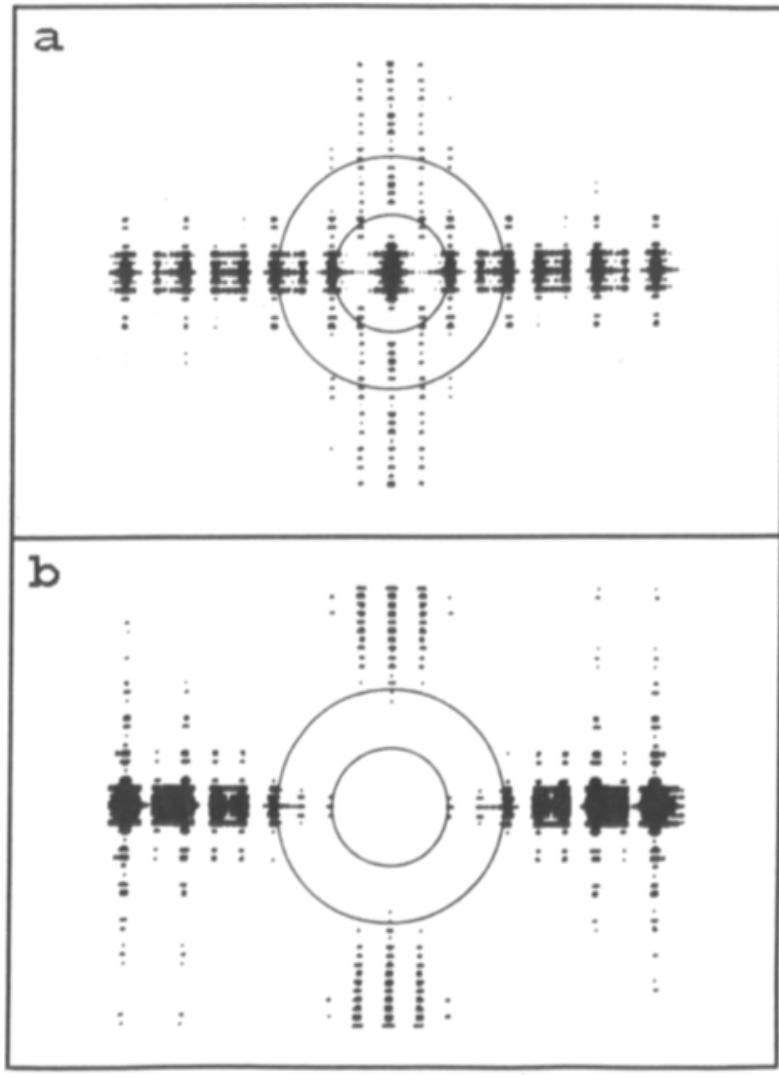

Figure 3. Two-dimensional Fourier transformations of the stimuli shown in Figure 2. Figure 3a shows the transform for the bright stimulus shown in Figure 2a. Figure 3b shows the transform for the contrast-balanced stimulus shown in Figure 2b. The transforms are plotted in polar coordinates, with spatial frequency on the radial dimension and orientation on the angular dimension. Spatial frequency increases linearly from zero at the center. The radii of the inner and outer circles are at 3 and 6 cycles/degree, respectively. The horizontal meridian of the polar coordinates indicates horizontal spatial frequency components and the vertical meridian indicates vertical spatial frequency components. The size of each plotted point indicates the power of a given spatial frequency at a given orientation. When stimuli contain substantial power at many similar spatial frequencies and orientations, plotted points overlap and produce larger filled areas. 
straint. Each trial began with a 500 -msec tone followed immediately by a 500 -msec presentation of the central fixation point. The subjects were instructed to look directly at the fixation point and not to move their eyes. The fixation point was followed immediately by a 100 -msec presentation of one of the stimulus patterns, which appeared randomly, but equally often, above or below the fixation point $\left(1.2^{\circ}\right.$ from fixation to the nearest edge of the stimulus pattern). There was a 1 -sec ITI.

The subjects used their dominant hand to indicate which of the target letters (" $H$ " or " $S$ ") was present on each trial. Right-handed subjects pressed the " $\mathrm{H}$ " key with their index finger and the " $\mathrm{S}$ " key with their middle finger. Left-handed subjects had the opposite mapping. The subjects were instructed to respond as quickly as possible while minimizing errors.

Each subject received four blocks of 67 trials. The first 3 trials of each block were considered warm-up trials and were not included in the analysis. Half the subjects received two blocks (one local bias and one global bias) with bright stimuli followed by two blocks with contrast-balanced stimuli, and this order was reversed for the other half. For local bias blocks, $75 \%$ of the targets appeared at the local level and 25\% appeared at the global level. For global bias blocks, these probabilities were reversed. The subjects were not informed of the changing target probabilities associated with the different blocks. The order of the local bias and global bias blocks was counterbalanced between subjects, but a given subject received the same order for both types of stimuli. Target letter (" $\mathrm{H}$ " and " $S$ "), distractor letter ("A" and " $E$ "), and stimulus location (above and below fixation) were completely counterbalanced within each block. The stimuli were presented randomly, except that the target could not be the same letter, or appear in the same location, on more than four consecutive trials. In addition, the target appeared at the biased level for at least the first 8 trials of each block in an attempt to draw attention to that level. The subjects received a practice block of 16 bright stimuli before the two bright data blocks and a second practice block of 16 contrast-balanced stimuli before the two contrast-balanced data blocks. Targets occurred equally often at the local and global levels in both practice blocks.

\section{Results}

The data (both RTs and errors) were subjected to an analysis of variance (ANOVA) with stimulus type (bright or contrast-balanced), bias level (local and global), target level (local and global), target letter (" $H$ "' and ' $S$ '), and distractor letter (" $A$ " and " $E$ ") as repeated measures factors. Median RTs (correct trials only) were calculated for each cell in the design for each subject, and the RT data reported are means of those medians. Mean error rates and $\mathrm{RT}$ s for each cell in the design are given in $\mathrm{Ta}-$ bles 1 and 2, respectively.

Errors. There was a significant bias level $\times$ target level interaction $[F(1,11)=34.45, p<.001]$, reflecting the fact that subjects made fewer errors to local than to global targets in the local bias condition but fewer errors to global than to local targets in the global bias condition. The target letter $\times$ distractor letter interaction was also significant $[F(1,11)=14.70, p<.01]$, reflecting the fact that subjects made fewer errors for " $H$ " than for " $S$ " targets if the distractor was an " $A$," but fewer errors for " $S$ " than for " $H$ "' targets if the distractor was an " $E$.", Finally, this was the case for local but not for global targets (asymmetric global interference), as indicated by a significant target letter $\times$ distractor letter $\times$ target level interaction $[F(1,11)=18.70, p<.001]$. The same effects were also present in the RT data.

Response time: Biasing. As shown in Figure 4, the bias level $\times$ target level interaction was significant $[F(1,11)=34.09, p<.001]$, reflecting the fact that RTs were faster to local targets than to global targets in the local bias condition $[F(1,11)=23.74, p<.001]$, but faster to global targets than to local targets in the global

Table 1

Experiment 1 Percentage Errors for Each Target and Distractor Letter as a Function of Stimulus Type and Bias Level

\begin{tabular}{|c|c|c|c|c|c|c|c|c|c|}
\hline \multirow[b]{3}{*}{ Stimulus Type } & \multirow[b]{3}{*}{ Bias } & \multicolumn{8}{|c|}{ Target } \\
\hline & & \multicolumn{2}{|c|}{ Global H } & \multicolumn{2}{|c|}{ Global S } & \multicolumn{2}{|c|}{ Local $\mathrm{H}$} & \multicolumn{2}{|c|}{ Local S } \\
\hline & & A & $\mathrm{E}$ & A & $\mathrm{E}$ & A & $\mathrm{E}$ & $\mathrm{A}$ & $\bar{E}$ \\
\hline & global & 2.1 & 3.5 & 3.5 & 3.5 & 8.3 & 14.6 & 10.4 & 4.2 \\
\hline \multirow{2}{*}{ Bright } & local & 12.5 & 4.2 & 8.3 & 6.3 & 2.8 & 6.3 & 4.9 & 1.4 \\
\hline & global & 5.6 & 2.8 & 4.2 & 4.9 & 4.2 & 22.9 & 20.8 & 2.1 \\
\hline Contrast-balanced & local & 12.5 & 10.4 & 16.7 & 4.2 & 2.8 & 4.9 & 10.4 & 0.7 \\
\hline
\end{tabular}

Note $-A$ and $E$ are distractors.

Table 2

Experiment 1 Response Times (in Milliseconds) for Each Target and Distractor Letter as a Function of Stimulus Type and Bias Level

\begin{tabular}{|c|c|c|c|c|c|c|c|c|c|}
\hline \multirow[b]{3}{*}{ Stimulus Type } & \multirow[b]{3}{*}{ Bias } & \multicolumn{8}{|c|}{ Target } \\
\hline & & \multicolumn{2}{|c|}{ Global $\mathrm{H}$} & \multicolumn{2}{|c|}{ Global S } & \multicolumn{2}{|c|}{ Local H } & \multicolumn{2}{|c|}{ Local S } \\
\hline & & $\mathrm{A}$ & $\mathrm{E}$ & A & $\mathbf{E}$ & A & $E$ & A & $\mathrm{E}$ \\
\hline \multirow{3}{*}{ Bright } & global & 521 & 492 & 588 & 569 & 627 & 772 & 756 & 620 \\
\hline & local & 633 & 567 & 710 & 705 & 508 & 526 & 550 & 493 \\
\hline & global & 602 & 571 & 664 & 646 & 596 & 722 & 686 & 617 \\
\hline Contrast-balanced & local & 688 & 715 & 714 & 692 & 498 & 528 & 586 & 515 \\
\hline
\end{tabular}

Note- $\mathrm{A}$ and $\mathrm{E}$ are distractors. 


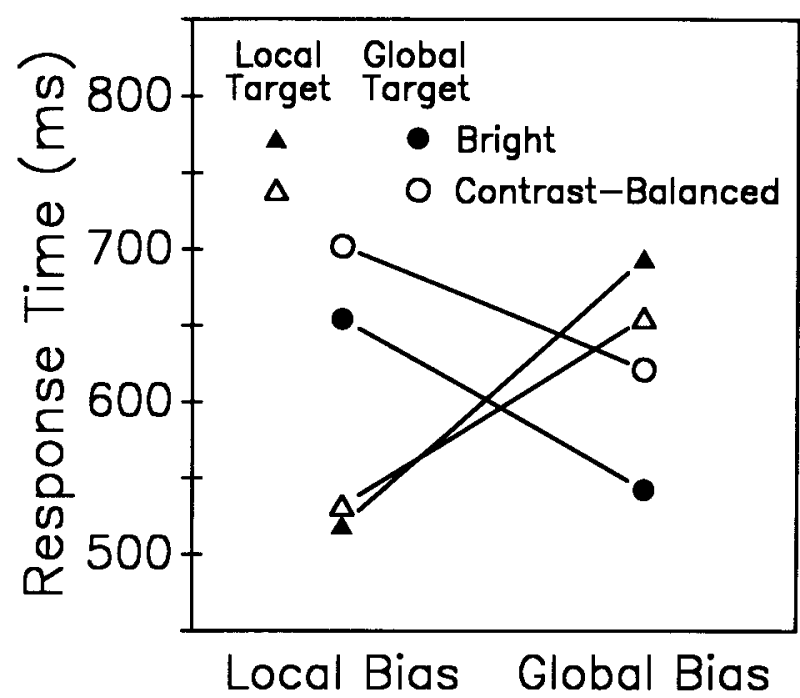

Figure 4. Experiment 1: Response time (in milliseconds) to global (circles) and local (triangles) targets for the bright (filled symbols) and contrast-balanced (open symbols) stimuli, as a function of biasing condition.

bias condition $[F(1,11)=16.17, p<.01]$. Thus, biasing produced the expected performance tradeoff taken to be indicative of attentional shifts in response to the changing task demands. The RTs were also faster in the local bias condition than in the global bias condition $[F(1,11)=$ $4.92, p<.05]$.

There was little suggestion that the ability to shift attention between local and global forms was based on selection between high versus low spatial frequency channels. Eliminating low spatial frequencies affected biasing very little. The bias level $\times$ target level interaction was significant for both bright stimuli $[F(1,11)=24.29, p<$ $.001]$ and contrast-balanced stimuli $[F(1,11)=37.63$, $p<.001]$. The stimulus type $\times$ bias level $\times$ target level interaction was nonsignificant $[F(1,11)=3.4]$.

Although contrast balancing had little effect on attentional shifts between local and global forms, it did slow RTs to global forms overall (see Figure 4). There was a significant stimulus type $\times$ target level interaction $[F(1,11)=12.16, p<.01]$, reflecting the fact that contrast balancing slowed RTs to global $[F(1,11)=7.68$, $p<.05]$, but not to local, forms $(F<1)$. This is perhaps not surprising, since contrast balancing eliminates low spatial frequencies that specify global forms while leaving intact high spatial frequencies that specify local forms. These data suggest that the global RT advantage so frequently observed in the literature depends on low spatial frequency information.

Interference. The target letter $\times$ distractor letter interaction was significant $[F(1,11)=34.66, p<.001]$, reflecting the fact that RTs were faster for " $\mathrm{H}$ " than for " $S$ " targets if the distractor was an " $A$," but faster for
" $S$ " than for " $H$ " targets if the distractor was an " $E$." Thus, as expected, distractor letters influenced RTs to target letters. In addition, this was the case for local but not for global targets (asymmetric global interference), as indicated by a significant target level $\times$ target letter $\times$ distractor letter interaction $[F(1,11)=23.79, p<.001]$.

Biasing and interference. As can be seen in Figure 5, asymmetric global interference was relatively immune to variations in attention. In the global bias condition, RTs were faster to global than to local targets $[F(1,11)=$ $16.17, p<.01]$, and there was a target letter $\times$ distractor letter interaction for responding to local $[F(1,11)=$ $34.63, p<.001]$, but not to global $(F<1)$, targets. Likewise in the local bias condition, there was still a target letter $\times$ distractor letter interaction for responding to local $[F(1,11)=10.11, p<.01]$, but not for responding to global $(F<1)$, targets. This occurred despite the fact that the RT advantage was reversed in the local bias condition $[F(1,11)=23.74, p<.001]$. Thus asymmetric global interference occurred not only when there was a global RT advantage (as predicted by the precedence account), but also when there was a local RT advantage (which is not consistent with the precedence account).

Interference was not wholly insensitive to variations in attention, however. The bias level $\times$ target level $\times$ target letter $\times$ distractor letter interaction was significant $[F(1,11)=8.49, p<.05]$, reflecting the fact that asymmetric global interference was more pronounced in the global bias than in the local bias condition. Nevertheless, these data show that RT advantage and interference cannot simply reflect precedence. Interference and RT ad-

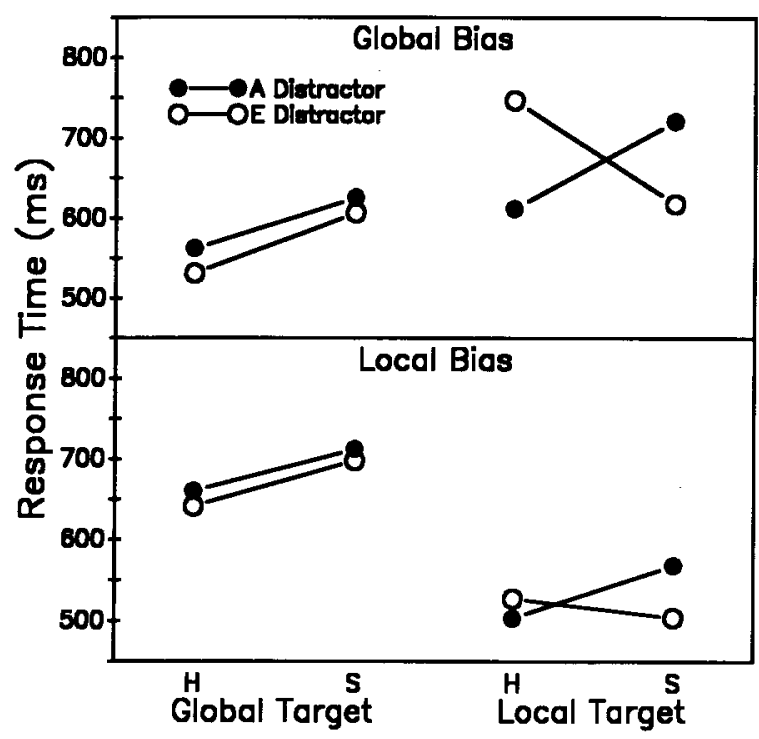

Figure 5. Experiment 1: Response time (in milliseconds) for global and local " $H$ " and " $S$ " targets when the distractor letter was an " $A$ " (closed symbols) or an " $\mathrm{E}$ " (open symbols), as a function of biasing condition. 


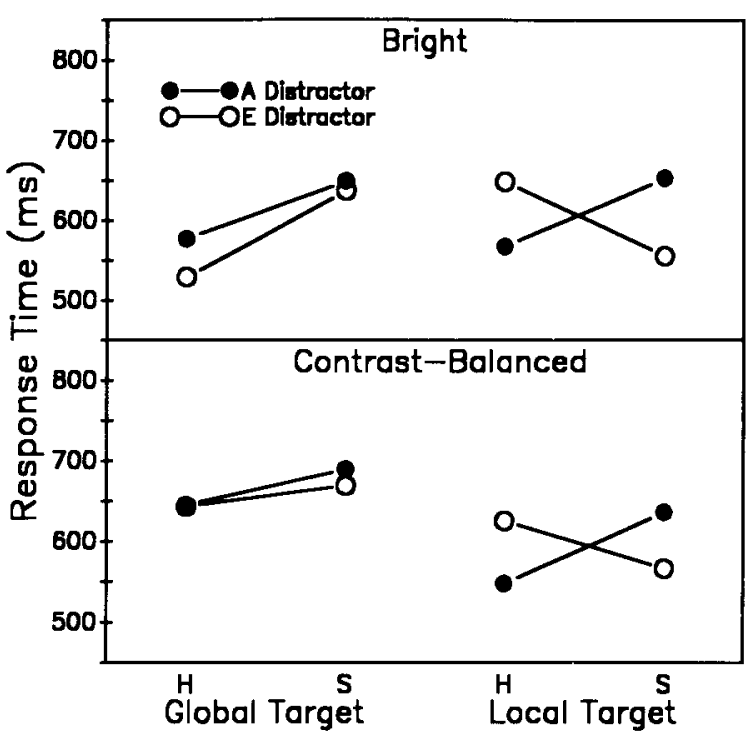

Figure 6. Experiment 1: Response time (in milliseconds) for global and local " $\mathrm{H}$ " and " $S$ " targets when the distractor letter was an " $A$ " (closed symbols) or an " $E$ " (open symbols), as a function of stimulus type.

vantage did not covary as they should have if that were the case.

Contrast balancing and interference. Interference was also relatively immune to variations in spatial frequency (see Figure 6). The target level $\times$ target letter $\times$ distractor letter interaction was significant for both bright stimuli $[F(1,11)=16.30, p<.01]$ and contrast-balanced stimuli $[F(1,11)=15.38, p<.01]$. Thus, asymmetric global interference occurred even when low spatial frequencies were eliminated. On the other hand, contrast balancing did have a small effect on interference. The stimulus type $\times$ bias level $\times$ target letter $\times$ distractor letter interaction was significant $[F(1,11)=5.79, p<.05]$. This interaction occurred because the target letter $\times$ distractor letter interaction was greater in the global bias than in the local bias condition when the stimuli were bright $[F(1,11)=6.93, p<.05]$, but not when they were contrast balanced $(F<1)$. Finally, RTs were faster to " $\mathrm{H}$ " than to "S" targets $[F(1,11)=5.06, p<.05]$. This difference was more pronounced for global than for local targets $[F(1,11)=14.25, p<.01]$, especially for bright stimuli $[F(1,11)=6.82, p<.05]$.

\section{Discussion}

The results of Experiment 1 show that low spatial frequencies facilitate the processing of global forms. Eliminating low spatial frequencies by contrast balancing slowed RTs to global but not to local forms. This suggests that the frequently reported RT advantage for global forms reflects a temporal processing advantage provided by low spatial frequencies. On the other hand, several other proposed roles for spatial frequency channels in the analysis of hierarchical stimuli were not supported by the results of Experiment 1.

These data lend no support to the hypothesis that interference between local and global forms results from inhibitory interactions between high and low spatial frequency channels. There was asymmetric global interference even for contrast-balanced stimuli that would not have activated low spatial frequency channels. These data also provide little support for the hypothesis that interference between local and global forms reflects precedence of low versus high spatial frequency information. First, if interference reflects precedence, contrast balancing should have changed the pattern of interference effects. Eliminating low spatial frequencies, and thereby any global precedence derived from low spatial frequency channels, should also eliminate asymmetric global interference. However, asymmetric global interference occurred with both bright and contrast-balanced stimuli. This finding is tempered somewhat by the fact that interference was slightly reduced for contrast-balanced stimuli (see the right side of Figure 6), which suggests that interference may depend partially on low frequency precedence. However, even for the broadband bright stimuli, interference did not covary with RT advantage as it should have if these measures reflected precedence. There was asymmetric global interference (consistent with low frequency precedence) even in the local bias condition, which produced a local RT advantage (consistent with high frequency precedence). Thus the present data suggest that interference between local and global forms depends little, if at all, on the functional characteristics of spatial frequency channels.

The present results also provide little support for the idea that attentional selection between local and global forms depends on spatial frequency. The performance tradeoff produced by biasing did not differ for bright and contrast-balanced stimuli even though both local and global letters would have to be identified on the basis of high spatial frequency information for the contrastbalanced stimuli. These data show that some mechanism other than selection between high and low spatial frequency channels is sufficient to produce performance tradeoffs between local and global forms.

On the other hand, there was a nonsignificant trend toward attenuated biasing for the contrast-balanced stimuli. Global RTs were $112 \mathrm{msec}$ faster in the global bias condition than in the local bias condition for bright stimuli, but only $81 \mathrm{msec}$ faster for contrast-balanced stimuli. Likewise, local RTs were $175 \mathrm{msec}$ faster in the local bias condition than in the global bias condition for bright stimuli, but only $123 \mathrm{msec}$ faster for contrastbalanced stimuli. This raises the possibility that spatial frequency played a small but real role in shifting attention between local and global forms that Experiment 1 was not sensitive enough to detect.

In addition, the procedure used in Experiment 1 may not have been optimal for inducing selection by spatial frequency. In Experiment 1, stimuli appeared randomly above or below fixation within each block of trials. This 
locational uncertainty may have hindered selection by spatial frequency because there is good reason to believe that spatial frequency channels are spatially selective (R. L. De Valois \& K. K. De Valois, 1990). It might be, then, that evidence supporting selection by spatial frequency would have been found if stimuli had appeared at a fixed location.

\section{EXPERIMENT 2}

The procedure of Experiment 2 was identical to that of Experiment 1, except that the stimuli in Experiment 2 occurred at exactly the same location for every trial within a block, and the visual angle of the stimulus patterns was reduced. The latter change was introduced to reduce the error rate, which was relatively high in Experiment 1 for some cells of the design (see Table 1). In Experiment 1, the near edge of each stimulus pattern appeared $1.2^{\circ}$ from fixation. This meant that the far edge extended $6.3^{\circ}$ into the periphery. In Experiment 2, the near edge of each stimulus pattern was maintained at $1.2^{\circ}$ from fixation but the visual angle of the pattern was reduced so that the far edge extended only $4.4^{\circ}$ into the periphery.

\section{Method}

Subjects. The 5 male and 11 female subjects ranged in age from 17 to 59 years $(M=32.6, S D=12.1)$ and had the characteristics specified in Experiment 1 except that 2 were left handed. Five subjects ( 2 male and 3 female) also served as subjects in Experiment 1.

Apparatus, Stimuli, and Procedure. All aspects of the apparatus and stimuli for Experiment 2 were identical to those in Experiment 1 , except that the stimuli subtended a smaller visual angle. Global letters were $3.2^{\circ}$ high and $2.0^{\circ}$ wide. Local letters were $.44^{\circ}$ high and $.27^{\circ}$ wide. This change altered the absolute but not the relative spatial frequencies of local and global forms.
The procedure for Experiment 2 was also the same as that for Experiment 1, except that the location of the stimuli above or below fixation was held constant within each block of trials. Each subject in Experiment 2 went through the entire sequence of practice and data blocks from Experiment 1 twice, once with all stimuli appearing above fixation and once with all stimuli appearing below fixation. The order of presentation of stimuli above and below fixation was counterbalanced between subjects.

\section{Results and Discussion}

The data for Experiment 2 were subjected to the same repeated measures ANOVA that was used for Experiment 1. Error and RT data for Experiment 2 are presented in Tables 3 and 4, respectively.

Errors. As expected, errors (and RTs) were lower in Experiment 2 than in Experiment 1 but the pattern was much the same (compare Tables 1 and 3 ). Biasing produced the expected performance tradeoff between local and global targets, as was indicated by a significant bias level $\times$ target level interaction $[F(1,15)=17.32, p<.001]$. The expected interference effects also occurred, producing significant target letter $\times$ distractor letter $[F(1,15)=10.33$, $p<.01]$ and target letter $\times$ distractor letter $\times$ target level $[F(1,15)=7.13, p<.05]$ interactions.

Response time: Biasing. The biasing effects in Experiment 2 replicated those of Experiment 1 (see Figure 7). The bias level $\times$ target level interaction was significant $[F(1,15)=33.64, p<.001]$, reflecting the fact that RTs were faster to local targets than to global targets in the local bias condition $[F(1,15)=22.89, p<.001]$, but faster to global targets than to local targets in the global bias condition $[F(1,15)=17.84, p<.001]$.

As in Experiment 1, eliminating low spatial frequencies had little effect on biasing. The bias level $\times$ target

Table 3

Experiment 2 Percentage Errors for Each Target and Distractor Letter as a Function of Stimulus Type and Bias Level

\begin{tabular}{|c|c|c|c|c|c|c|c|c|c|}
\hline \multirow[b]{3}{*}{ Stimulus Type } & \multirow[b]{3}{*}{ Bias } & \multicolumn{8}{|c|}{ Target } \\
\hline & & \multicolumn{2}{|c|}{ Global H } & \multicolumn{2}{|c|}{ Global S } & \multicolumn{2}{|c|}{ Local H } & \multicolumn{2}{|c|}{ Local S } \\
\hline & & A & $\mathbf{E}$ & A & $\mathrm{E}$ & A & $\mathrm{E}$ & A & $\mathrm{E}$ \\
\hline \multirow{3}{*}{ Bright } & global & 2.6 & 0.3 & 4.5 & 1.8 & 1.6 & 7.0 & 7.0 & 4.7 \\
\hline & local & 3.1 & 1.6 & 4.7 & 5.5 & 0.5 & 2.9 & 2.6 & 2.1 \\
\hline & global & 1.8 & 1.8 & 3.1 & 2.6 & 0.8 & 3.9 & 14.8 & 2.3 \\
\hline Contrast-balanced & local & 5.5 & 9.4 & 4.7 & 7.0 & 0.5 & 2.3 & 1.8 & 1.3 \\
\hline
\end{tabular}

Note $-\mathbf{A}$ and $\mathbf{E}$ are distractors.

Table 4

Experiment 2 Response Times (in Milliseconds) for Each Target and Distractor Letter as a Function of Stimulus Type and Bias Level

\begin{tabular}{|c|c|c|c|c|c|c|c|c|c|}
\hline \multirow[b]{3}{*}{ Stimulus Type } & \multirow[b]{3}{*}{ Bias } & \multicolumn{8}{|c|}{ Target } \\
\hline & & \multicolumn{2}{|c|}{ Global H } & \multicolumn{2}{|c|}{ Global S } & \multicolumn{2}{|c|}{ Local H } & \multicolumn{2}{|c|}{ Local S } \\
\hline & & A & $\mathrm{E}$ & A & $\mathrm{E}$ & A & $\mathbf{E}$ & A & $\mathrm{E}$ \\
\hline \multirow{3}{*}{ Bright } & global & 486 & 462 & 548 & 510 & 482 & 632 & 664 & 568 \\
\hline & local & 540 & 549 & 630 & 590 & 448 & 490 & 509 & 480 \\
\hline & global & 505 & 501 & 575 & 578 & 534 & 579 & 656 & 542 \\
\hline Contrast-balanced & local & 551 & 587 & 646 & 614 & 456 & 476 & 502 & 492 \\
\hline
\end{tabular}

Note $-A$ and $E$ are distractors. 


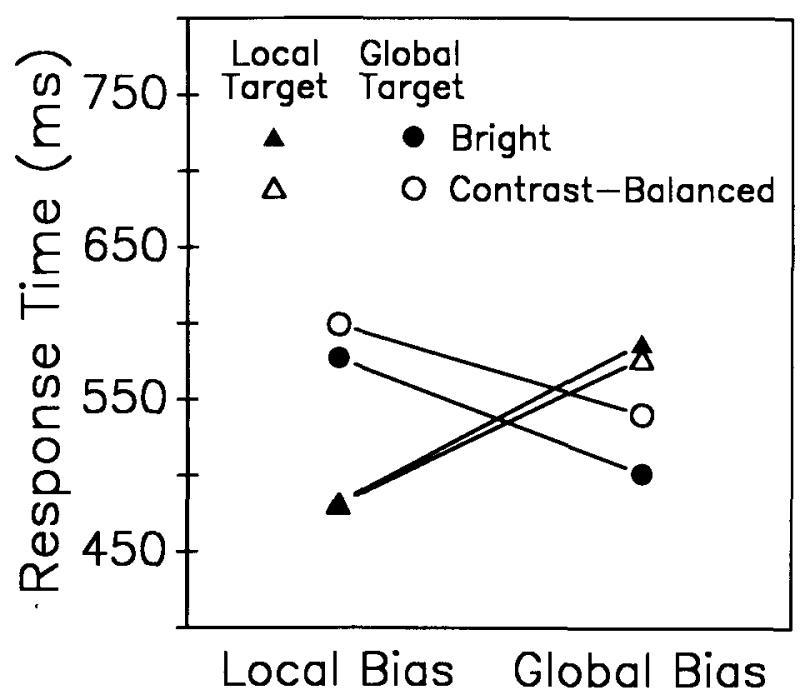

Figure 7. Experiment 2: Response time (in milliseconds) to global (circles) and local (triangles) targets for the bright (filled symbols) and contrast-balanced (open symbols) stimuli, as a function of biasing condition.

level interaction was significant for both bright stimuli $[F(1,15)=28.65, p<.001]$ and contrast-balanced stimuli $[F(1,15)=28.54, p<.001]$. The stimulus type $\times$ bias level $\times$ target level interaction was nonsignificant $[F(1,15)=1.03]$.

Again replicating Experiment 1, contrast balancing slowed RTs to global $[F(1,15)=8.42, p<.05]$, but not to local, forms $(F<1)$. This difference was confirmed by a significant stimulus type $\times$ target level interaction $[F(1,15)=6.26, p<.05]$.

Recall that in Experiment 1 there was a nonsignificant trend toward attenuated biasing for the contrast-balanced stimuli. A similar, though smaller, effect was evident in Experiment 2 as well. Global RTs were 76 msec faster in the global bias condition than in the local bias condition for bright stimuli, but only $59 \mathrm{msec}$ faster for contrast-balanced stimuli. Likewise, local RTs were $105 \mathrm{msec}$ faster in the local bias condition than in the global bias condition for bright stimuli, but only $96 \mathrm{msec}$ faster for contrast-balanced stimuli. Thus, mean RTs in both Experiments 1 and 2 suggest that attentional selection might be facilitated somewhat by differences in spatial frequency between local and global forms. However, in both experiments, the effects were small and statistically nonsignificant. At least under present conditions, then, differences in spatial frequency between local and global forms does little, if anything, to facilitate attentional selection.

Interference. In Experiment 2, as in Experiment 1, there was asymmetric global interference. The target letter $\times$ distractor letter interaction was significant $[F(1,15)=$ $23.0, p<.001]$, as was the target level $\times$ target letter $\times$ distractor letter interaction $[F(1,15)=13.74, p<.01]$.
Biasing and interference. In the global bias condition, RTs were faster to global than to local targets $[F(1,15)=$ $17.84, p<.001]$, and there was also a target letter $\times$ distractor letter interaction for responding to local $[F(1,15)=27.62, p<.001]$, but not to global $(F<1)$, targets (see Figure 8 ). In addition, despite the fact that the RT advantage was reversed in the local bias condition $[F(1,15)=22.89, p<.001]$, there was a target letter $\times$ distractor letter interaction for responding to local $[F(1,15)=22.24, p<.001]$, but not global, targets. $\mathrm{Fi}_{-}$ nally, the bias level $\times$ target level $\times$ target letter $\times$ distractor letter interaction was significant $[F(1,15)=17.28$, $p<.001]$, reflecting the fact that asymmetric global interference was more pronounced in the global bias than in the local bias condition.

This set of findings is identical to those of Experiment 1 . However, there is some suggestion that biasing had more of an effect on interference in Experiment 2 than in Experiment 1 (compare Figures 5 and 8). Visual inspection of the data indicates that local biasing not only reduced interference for responding to local forms, but also (unlike in Experiment 1) established interference for responding to global forms. However, the target letter $x$ distractor letter interaction when responding to global targets in the local bias condition was nonsignificant for Experiment $2[F(1,15)=3.27, p<.09]$. On the other hand, the same effect produced an $F<1$ in Experiment 1, suggesting the possibility that biasing may have had more of an effect on interference in Experiment 2 than in Experiment 1 . Even so, the results of Experiment 2 replicate those of Experiment 1 in showing that asymmetric global interference can occur even when there is a local RT advantage.

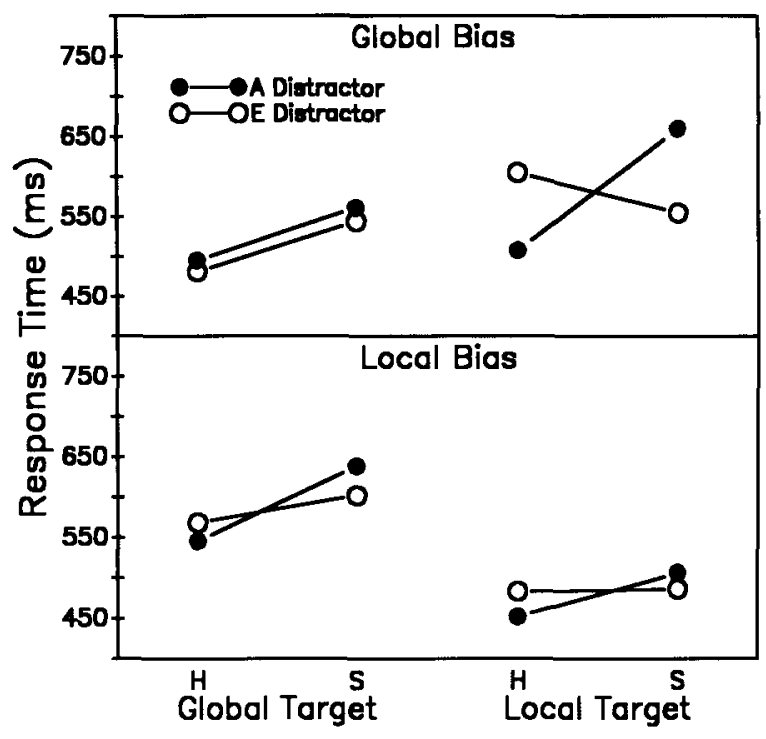

Figure 8. Experiment 2: Response time (in milliseconds) for global and local " $H$ " and " $S$ " targets when the distractor letter was an " $A$ " (closed symbols) or an " $\mathrm{E}$ " (open symbols), as a function of biasing condition. 


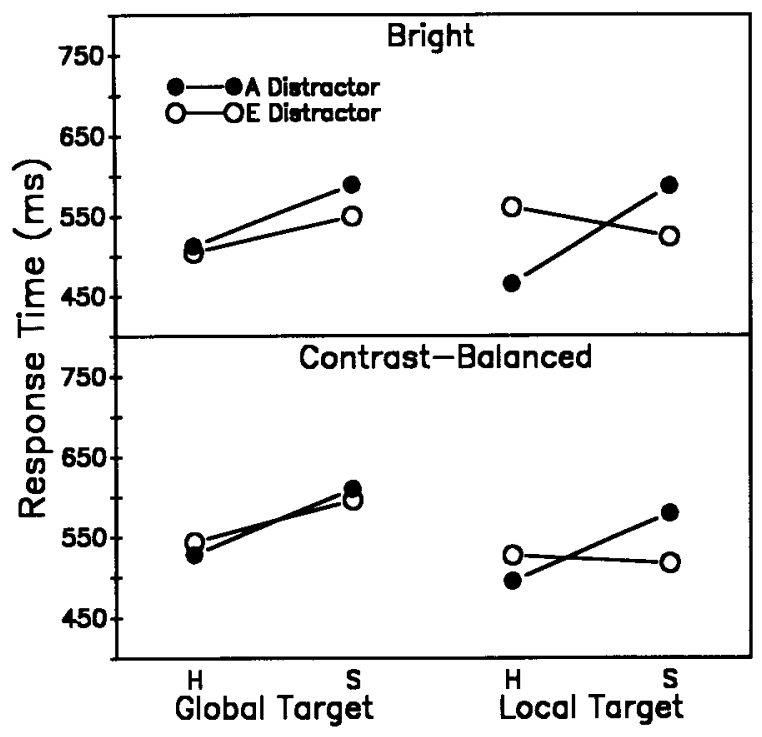

Figure 9. Experiment 2: Response time (in milliseconds) for global and local " $H$ " and " $S$ " targets when the distractor letter was an " $A$ " (closed symbols) or an " $\mathrm{E}$ " (open symbols), as a function of stimulus type.

Contrast balancing and interference. Again replicating the results of Experiment 1, interference was relatively immune to variations in spatial frequency (see Figure 9). The target level $\times$ target letter $\times$ distractor letter interaction was significant for both bright stimuli $[F(1,11)=$ 23.26, $p<.001]$ and contrast-balanced stimuli $[F(1,11)=$ $4.61, p<.05]$. Thus, asymmetric global interference occurred even for stimuli that had no low spatial frequency content. On the other hand, contrast balancing did have some effect on interference. The stimulus type $\times$ target level $\times$ target letter $\times$ distractor letter interaction was also significant $[F(1,15)=7.24, p<.05]$, reflecting the fact that asymmetric global interference was more pronounced for bright than for contrast-balanced stimuli. Finally, RTs were faster to " $H$ " than to " $S$ " targets $[F(1,15)=20.02, p<.001]$.

\section{GENERAL DISCUSSION}

In both of the present experiments, contrast balancing slowed RTs to global forms. This finding suggests that the global RT advantage frequently reported in the literature might result from a temporal advantage conferred by low spatial frequency information. On the other hand, the present experiments provided little support for a more extensive role of spatial frequency in the analysis of local and global levels of structure.

The present data lend no support to the hypothesis that asymmetric global interference results from the inhibition of high spatial frequency channels by low spatial frequency channels. There was asymmetric global interference even for the contrast-balanced stimuli in both Experiments 1 and 2. That is, global forms interfered with the analysis of local forms even for stimuli that could not have activated low spatial frequency channels. This is consistent with the data provided by Hughes et al. (1990), who also found interference between local and global forms with contrast-balanced stimuli. It is not clear why filtering low spatial frequencies eliminated interference in the experiment by Badcock et al. (1990). There were many procedural differences among the different experiments. However, the fact that interference does not occur under all conditions (Badcock et al., 1990) does not alter the fact that interference between local and global forms can occur even in the absence of interactions between low and high spatial frequency channels (Hughes et al., 1990; and the present experiments).

The present results are also not easily reconciled with the hypothesis that interference reflects precedence. This is true, regardless of whether precedence is viewed in terms of hierarchical structure or in terms of spatial frequency. There was asymmetric global interference (consistent with global or low frequency precedence) even in the local bias condition in which there was a local RT advantage (consistent with local or high frequency precedence). Thus, interference did not covary with RT advantage as it should have if these measures both reflected precedence. This was the case for both bright and contrastbalanced stimuli.

It has recently been suggested that interference might reflect a process of perceptual integration of information from different levels of structure (Lamb et al., 1989, 1990; Robertson \& Lamb, 1991). Recent neuropsychological evidence supports this view. Several studies have provided evidence that the left hemisphere is biased toward the analysis of local levels of structure, whereas the right hemisphere is biased toward the analysis of global levels of structure (Delis, Robertson, \& Efron, 1986; Doyon \& Milner, 1991; Lamb et al., 1989, 1990; Robertson et al., 1988). Furthermore, brain lesions that disrupt communication between the hemispheres eliminate interference (Humphreys, Riddoch, \& Quinlan, 1985; Lamb et al., 1989, 1990; Robertson, Lamb, \& Zaidel, 1993). These data have led to the suggestion that information about different levels of structure is analyzed in parallel and then integrated via the sharing of information between the hemispheres. When this integration process is interrupted via injury to the brain, interference is eliminated. Since this hypothesis does not assume that interference reflects precedence, the lack of covariation between RT advantage and interference in the present studies poses no problem.

The present experiments also provide little support for the idea that attentional selection between local and global forms is based on spatial frequency. Eliminating low spatial frequencies from the stimulus had no measurable effect on shifts of attention between local and global forms. Thus, selection can occur on some basis other than spatial frequency. Of course, the possibility remains that selection on the basis of spatial frequency might occur under some conditions. However, the present data show 
that little, if anything, is gained by having spatial frequency available as a potential basis for selection, at least when other cues are available.

As already noted, it has been argued that changes in the diameter or location of an attentional "spotlight" can differentially affect RTs to local and global forms (Lamb \& Robertson, 1988, 1990; Ward, 1982). The contribution of this type of selection mechanism should have been minimized in Experiment 1, because the location of the stimuli varied randomly. However, although this would prevent subjects from gaining an advantage by simply attending to a given location, it would not prevent them from adopting a relatively small diameter spotlight in local bias blocks and a relatively large diameter spotlight in global bias blocks. The spatial certainty of the stimuli in Experiment 2 would be even more conducive to this type of mechanism, because the spotlight could be optimized in terms of location as well as size. Thus, it is possible that the performance tradeoffs in the present experiments were produced by variations in the diameter and/or location of an attentional spotlight.

The present data also are not inconsistent with the suggestion that the analysis of local and global forms is affected by a "categorical" selection mechanism as well as by an attentional spotlight (Lamb \& Robertson, 1990; Robertson, Egly, Lamb, \& Kerth, 1993). The idea here is that attributes associated with local versus global forms (e.g., high vs. low spatial frequencies) might be assigned to different categories and that selection occurs by differentially weighting the different categories, depending on task demands. However, although nothing in the present data preclude categorical selection, they do not support the suggestion that high versus low spatial frequencies form the basis for those categories.

In summary, the present data suggest that spatial frequency plays an important but limited role in the analysis of hierarchical stimuli. The low spatial frequencies normally associated with more global levels of structure do confer a temporal processing advantage. On the other hand, interference between different levels of structure does not seem to depend on either temporal precedence of low spatial frequency information or on inhibition of high-frequency channels by low-frequency channels. Further, the present data provide little support for the hypothesis that attentional selection between local and global forms is based on spatial frequency. Although the present data do not rule out the possibility that attentional selection between local and global forms might be based on spatial frequency under some conditions, they do show that selection is affected little, if at all, by eliminating spatial frequency as a potential cue.

\section{REFERENCES}

Badcock, J. C., Whitworth, F. A., Badcock, D. R., \& Lovegrove, W. J. (1990). Low-frequency filtering and the processing of localglobal stimuli. Perception, 19, 617-629.

BREITMEyer, B. G. (1975). Simple reaction time as a measure of the temporal response properties of transient and sustained channels. $\mathrm{Vi}$ sion Research, 15, 1411-1412.
Breitmeyer, B. G., \& GANZ, L. (1976). Implications of sustained and transient channels for theories of visual pattern masking, saccadic suppression, and information processing. Psychological Review, 83, 1-36.

CAMPBell, F. W., \& RoBson, J. G. (1968). Application of Fourier analysis to the visibility of gratings. Journal of Physiology, 197, 551-566.

Carison, C. R., Anderson, C. H., \& Moeller, J. R. (1980). Visual illusions without low spatial frequencies. Investigative Ophthalmology \& Visual Science, 19(Suppl.), 165-166.

Delis, D. C., Robertson, L. C. \& Erron, R. (1986). Hemispheric specialization of memory for visual hierarchical stimuli. Neuropsychologia, 24, 205-214.

DE VALOIS, K. K. (1977). Spatial frequency adaptation can enhance contrast sensitivity. Vision Research, 17, 1057-1065.

De Valois, R. L., \& De Valois, K. K. (1990). Spatial vision. New York: Oxford University Press.

DOYON, J., \& MILNER, B. (1991). Right temporal-lobe contribution to global visual processing. Neuropsychologia, 29, 343-360.

HARWERTH, R. S., \& LEVI, D. M. (1978). Reaction time as a measure of suprathreshold grating detection. Vision Research, 18, 1579-1586.

HuGHES, H. C. (1986). Asymmetric interference between components of suprathreshold compound gratings. Perception \& Psychophysics, 40, 241-250.

Hughes, H. C., Fendrich, R., \& Reuter-Lorenz, P. A. (1990). Global versus local processing in the absence of low spatial frequencies. Journal of Cognitive Neuroscience, 2, 272-282.

Hughes, H. C., Layton, W. M., Baird, J. C., \& Lester, L. S. (1984). Global precedence in visual pattern recognition. Perception \& Psychophysics, 35, 361-371.

Humphreys, G. W., RidDoCh, J., \& Quinlan, T. T. (1985). Interactive processes in perceptual organization: Evidence from visual agnosia. In M. I. Posner \& O. S. M. Marin (Eds.), Attention \& performance $X I$ (pp. 301-318). Hillsdale, NJ: Erlbaum.

Kinchla, R. A., Solis-Macias, V., \& Hoffman, J. (1983). Attending to different levels of structure in a visual image. Perception \& Psychophysics, 33, 1-10.

LAMB, M. R., \& RoberTson, L. C. (1987). Effects of acute alcohol on attention and the processing of hierarchical patterns. Alcoholism: Clinical \& Experimental Research, 11, 243-248.

LAMB, M. R., \& RoBertson, L. C. (1988). The processing of hierarchical stimuli: Effects of retinal locus, locational uncertainty, and stimulus identity. Perception \& Psychophysics, 44, 172-181.

LAmb, M. R., \& RoberTson, L. C. (1989). Do response time advantage and interference reflect the order of processing of global- and local-level information? Perception \& Psychophysics, 46, 254-258.

LAMB, M. R., \& RoBerTSON, L. C. (1990). The effect of visual angle on global and local reaction times depends on the set of visual angles presented. Perception \& Psychophysics, 47, 489-496.

LAMB, M. R., RoberTson, L. C., \& KNIGHT, R. T. (1989). Attention and interference in the processing of global and local information: Effects of unilateral temporal-parietal junction lesions. Neuropsychologia, 27, 471-483.

Lamb, M. R., Robertson, L. C., \& Knight, R. T. (1990). Component mechanisms underlying the processing of hierarchically organized patterns: Inferences from patients with unilateral cortical lesions. Journal of Experimental Psychology: Learning, Memory, \& Cognition, 16, $471-483$.

Navon, D. (1977). Forest before trees: The precedence of global features in visual perception. Cognitive Psychology, 9, 353-383.

NAvoN, D. (1991). Testing a queue hypothesis for the processing of global and local information. Journal of Experimental Psychology: General, 120, 173-189.

Navon, D., \& Norman, J. (1983). Does global precedence really depend on visual angle? Joumal of Experimental Psychology: Human Perception \& Performance, 9, 955-965.

PAQueT, L. (1992). Global and local processing in nonattended objects: A failure to induce local processing dominance. Journal of Experimental Psychology: Human Perception \& Performance, 18, 512-529.

Robertson, L. C., Egly, R., LAMb, M. R., \& Kerth, L. (1993). Spatial attention and cuing to global and local levels of hierarchical structure. Joumal of Experimental Psychology: Human Perception \& Performance, 19, 471-487.

Robertson, L. C., \& LAMB, M. R. (1991). Neuropsychological per- 
spectives on theories of part/whole organization. Cognitive Psychology, 23, 299-330.

Robertson, L. C., Lamb, M. R., \& KNight, R. T, (1988). Effects of lesions of temporal-parietal junction on perceptual and attentional processing in humans. Journal of Neuroscience, 8, 3757-3769

Robertson, L. C., Lamb, M. R., \& KNIGHT, R. T. (1991). Normal global-local analysis in patients with dorsolateral frontal lobe lesions. Neuropsychologia, 29, 959-967.

Robertson, L. C., LAMB, M. R., \& ZaideL, E. (1993). Interhemispheric relations in processing hierarchical patterns: Evidence from normal and commissurotomized subjects. Neuropsychology, 7, 325-342.

SERGENT, J. (1982). The cerebral balance of power: Confrontation or cooperation? Journal of Experimental Psychology: Human Perception \& Performance, 8, 253-272.

SERGENT, J. (1987). Failures to confirm the spatial-frequency hypothesis: Fatal blow or healthy complication? Canadian Journal of Psychology, 41, 412-428.

Shulman, G. L., Sullivan, M. A., Gish, K., \& Sakoda, W. J. (1986). The role of spatial-frequency channels in the perception of local and global structure. Perception, 15, 259-273.

Shulman, G. L., \& Wilson, J. (1987). Spatial frequency and selective attention to local and global information. Perception, 16, 89-101.

SINGER, W., \& BEDWORTH, N. (1973). Inhibitory interactions between $\mathrm{X}$ and $\mathrm{Y}$ units in the cat lateral geniculate nucleus. Brain Research, 49, 291-307.

TolHurst, D. J. (1972). Adaptation to square-wave gratings: Inhibition between spatial frequency channels in the human visual system. Journal of Physiology, 226, 231-249.
WARD, L. M. (1982). Determinants of attention to local and global features of visual forms. Journal of Experimental Psychology: Human Perception \& Performance, 8, 562-581.

\section{NOTE}

1. A less precise, but perhaps more intuitive way of verifying that low spatial frequencies were eliminated from the contrast-balanced stimuli is to consider the effect of viewing distance on performance. The spatial frequencies of a form increase with viewing distance. As viewing distance increases, the higher frequencies specifying local forms will begin to exceed the sensitivity range of the visual system, while lower frequencies specifying the global form will still be visible. In other words, at some distance, because of the low spatial frequency content of the typical hierarchical stimulus, an observer should be able to identify global but not local forms. This was the case for our bright stimuli. Using the first author as the observer, we found that at a viewing distance of $0.4 \mathrm{~m}$, global forms were identified correctly $100 \%$ of the time whereas local forms were identified at chance. In contrast, not only could contrastbalanced stimuli not be identified at that viewing distance, but performance was at chance for deciding whether or not a stimulus had even been presented. In other words, contrast-balanced stimuli were invisible from this distance. This shows that the bright stimuli contained low spatial frequencies sufficient to identify the global form that the contrastbalanced stimuli did not.

(Manuscript received August 28, 1992; revision accepted for publication May 24, 1993.) 